\title{
Review Paper on Energy- Efficient Protocols in Wireless Sensor Networks
}

\author{
Rajesh Chaudhary, Dr. Sonia Vatta \\ ${ }^{I}$ (STUDENT) CSE Department Bahra University, Shimla Hills Himachal Pradesh, INDIA \\ ${ }^{2}$ (GUIDE) CSE Department Bahra University, Shimla Hills Himachal Pradesh, INDIA
}

\begin{abstract}
A WSN is a specialized wireless network made up of large number of sensors and at least one base station. The foremost or the main difference between WSN and the traditional wireless networks is that sensors are extremely sensitive to energy consumption. Energy saving in the crucial issue in designing the wireless sensor networks [12]... Since the radio transmission and reception consumes more energy, one of the most or the main significant issue in wireless sensor network is the inherent limited battery power within network sensor nodes. It is preferable to dispense the energy throughout the wireless sensor network so to maximize the lifetime of sensor nodes. So it is essential to design effective and energy aware protocols in order to enhance the life time of the network. A wireless sensor network may have network structure based or protocol operation based routing protocol. In this paper, a review on hierarchical based routing protocol which is further a sub-type of the network structure based routing protocol in WSNs is carried out. Major issues which are considered in WSNs are Energy consumption and network life time.
\end{abstract}

Keywords: - Energy consumption, Wireless, Sensor network, routing protocols, energy efficient, network life time, sensor, battery, life.

\section{INTRODUCTION}

A wireless sensor network (WSN) are often outlined as a network consists of low-size and lowcomplex devices referred as sensor nodes that may sense the environment or surroundings and gather the knowledge from the observance field and communicate through wireless links; the information collected is forwarded, via multiple hops relaying to a sink (also referred to as controller or monitor) that may use it domestically, or is connected to alternative networks [2].

The sensor nodes are typically scattered in a sensor field as shown in Fig. 1. Every of those scattered sensor nodes has the capabilities to gather information and route information back to the sink and also the end users. Information is routed back to the end/finish user by multihop infrastructure design through the sink as shown in Fig. 1. The sink could communicate with the task manager node via web/internet or Satellite [3].

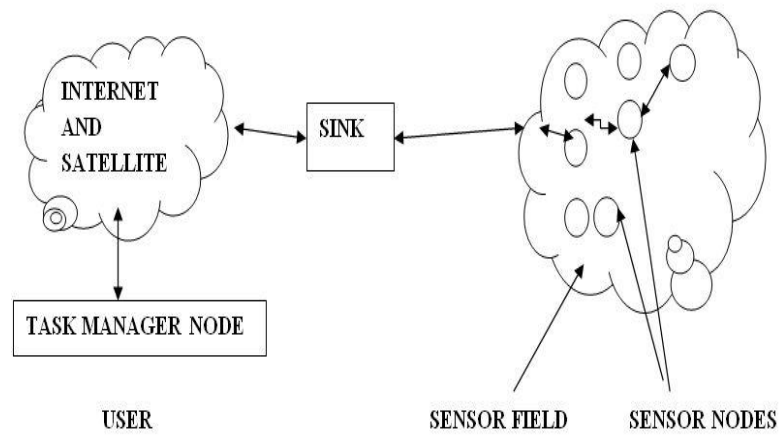

Fig: 1 Sensor nodes spreaded in a sensor field [9].

Designing protocols and applications for such networks needs to be energy aware so as to prolong the lifetime of the network, as a result of the substitution of the embedded batteries may be a terribly troublesome method once these nodes are put in or installed. Sensors usually link the physical world with the digital world by capturing and revealing real-world development and changing these into a kind that may be processed, stored, etc. Sensors offer remarkable or incredible benefits when integrated into varied devices, machines, and environments. [1]They will assist to avoid catastrophic infrastructure failures, conserve precious natural resources, boost productivity, improve security, and upgrade new applications such as context-aware systems and smart home technologies.

The miniaturisation of computing and sensing technologies allows the development of small, low-power, and cheap actuators, sensors, and controllers. 
Sensing is a very important technique that is used to gather information about a physical object or process including change in the state such as drop in temperature or pressure. The object that usually perform sensing task is termed as sensor. It consists of basic elements. Elements of sensor node are shown as below:

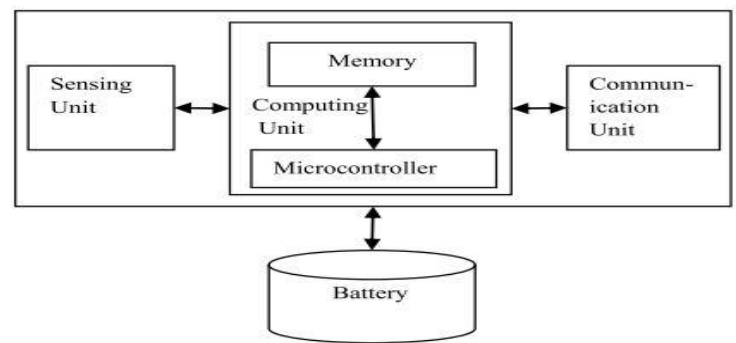

For example, the body of the human being is equipped with sensors that is they are able to capture optical data from the surroundings (eyes), acoustic information or data like sounds (ears), and smells (nose). These are the examples of remote sensors, which do not need to touch the monitored object to collect data. From a technical perspective, a sensor may be a device that translates parameters or events within the physical world into signals that may be measured and analyzed. Another unremarkably used term is transducer, which is usually used to describe a tool or device that converts energy from one kind into another [1].

When several sensors hand and glove monitor giant physical environments, then they usually outline a wireless sensor networks. Networks of many sensor nodes are usually wont to monitor giant geographic areas and statement environmental pollution and flooding, grouping structural health data on bridges using vibration sensors etc.

\section{WHY IT'S TO BE ENERGY EFFICIENT}

The most necessary constraint in or style challenge for wireless sensor network is energy potency. Sensor nodes are generally battery-powered through batteries that should be either replaced or recharged once depleted. Sensor nodes usually use the batteries for power provide within the wireless sensor networks. These sensor nodes are usually deployed or put in within the geographical area so as to watch the surroundings and to gather the knowledge from the geographical surroundings. Once the sensor nodes are deployed they are typically unapproachable to the operator. The vital or the foremost necessary point while using sensor nodes is that battery power ought to be consumed less so as to make wireless sensor network more energy economical or energy efficient. When these sensor nodes usually send the gather data or reports to the sink or base station they often consume the battery energy, due to which consumption of energy of the network will increases. Therefore energy conservation and energy economical or efficient routing protocol ought to be taken into consideration to develop the dynamic and adaptive idea within the networking for wireless sensor network. Designing an energy economical or efficient routing protocol that decrease the energy consumption of information transmissions and prolong the network life is a very important issue while creating wireless sensor network as energy economical or efficient.

\section{EXISTING HIERARCHICAL PROTOCOLS}

LEACH

LEACH is mostly called as Low Energy Adaptive Clustering Hierarchy protocol.

W.R. Heinzelman, A.P Chandrakasan and H. Balakrishnan [5] projected LEACH protocol in 2000. It is one of the mostly used hierarchical routing algorithms used in the sensor networks. The main plan of LEACH protocol is to divide the total wireless sensor network into many clusters. The cluster head node is randomly selected; the chance of every node to be selected as cluster head is equal attributable to which energy consumption of whole network is averaged. Thus LEACH will prolong the network life cycle.

Leach algorithm is cyclical; it provides a conception of round. Leach protocol runs with several rounds. Every round contains two states: cluster setup state and steady state. Within the cluster setup state it forms cluster within the self-adaptive mode whereas in steady state it transfers the information.

The selection of cluster head depends on decision made 0 and 1 . If number is smaller than the threshold value, the node becomes a cluster head for the current round. The threshold is mostly given as:

$\mathrm{T}(\mathrm{n})=\left\{\begin{array}{lr}\frac{\mathrm{p}}{1-\mathrm{p}^{*}\left(\mathrm{r}^{*} \bmod 1 / \mathrm{p}\right)} & \text { if } \mathrm{n} \varepsilon \mathrm{G} \\ 0 & \text { else }\end{array}\right.$

Where, $\mathrm{P}$ is that the desired percentage of cluster heads (e.g. is like $4 \%$ or $5 \%$ ), $\mathrm{r}$ is that the current round, and $\mathrm{G}$ is that the set of nodes that haven't been cluster heads within the last $1 / \mathrm{p}$ rounds. 
By considering this threshold, each node can be the cluster head at some point with $1 / \mathrm{p}$ rounds. Nodes that are cluster heads cannot become cluster head for the second time for $1 / \mathrm{p}-1$ rounds. Therefore, mostly every node has a $1 / p$ probability of becoming cluster in every round. At the end of each round, every traditional or normal node that's not a cluster head choose the closest or nearest cluster head and joins that cluster to transmit the information. The cluster heads mix or combine and compress the information and forward it to the sink or the base station, thus it extends the life of the most important or major nodes.

\section{Advantages in the LEACH protocol are:}

1. It is one of the mostly used hierarchical routing algorithms in sensor networks.

2. LEACH protocol erstwhile divides the total wireless sensor network into many clusters. Any node that served as a $\mathrm{CH}$ in present round cannot be selected as the $\mathrm{CH}$ again; therefore each node can share the load equally which is imposed on Cluster heads.

3. The cluster head node is randomly selected and chance of every node to be selected as cluster head is equal attributable to which energy consumption of whole network is averaged. Thus LEACH will prolong the network life cycle.

\section{Problems within the LEACH protocol are:}

1) The cluster head node is randomly selected in LEACH protocol. There are some shortcomings attributable to the likelihood of every node to be selected as cluster head is same. After numerous rounds, the node with or containing greater remaining energy and the node with smaller remaining energy have same likelihood or probability to be chosen as cluster head. If the node which has smaller remaining energy is chosen as cluster head, it'll run out of the energy and more die quickly, due to which network's robustness can be affected and life of the network become short.

2) The standard LEACH Protocol divides clusters randomly, additionally results in uneven distribution of clusters simply. Eventually the divided clusters might not be the simplest or best. As an example some clusters have a lot of nodes than others whereas some clusters have fewer nodes. Some cluster heads within the relatively central of clusters whereas some clusters heads may be in the edge of clusters far away from members. These phenomena will cause increase in energy consumption and make harsh impact on the total performance of the network.

3) In steady state, cluster head usually send information to the sink or base station directly, Cluster head that is farther from the sink communicate with the sink directly mostly spend a plenty of energy whereas transmission. Thus it'll crash earlier as a result of it runs out of energy. Particularly in the midst of the enlargement of the dimensions of the network, these effects are a lot of noticeable and have an effect on the network life seriously.

\section{PEGASIS}

S. Lindsey and C. Raghavendra [6] introduced Power Efficient Gathering in Sensor Information Systems (PEGASIS) protocol in 2002. It is an improved version of LEACH. Rather than forming clusters, it is based on forming chains of sensor nodes. One node is mainly responsible for routing the aggregated information to the sink. Every node aggregates the collected information with its own information, and then passes the aggregated data to the next ring. The distinction from LEACH is to employ or use multi hop transmission and choosing or selecting only one node to transmit to the sink or base station. Since the overhead caused by dynamic cluster formation is eliminated, multi hop transmission and data or information aggregation is employed or used, PEGASIS outperforms the LEACH.

\section{Advantages in the PEGASIS protocol are:}

1. It is an improved version of LEACH.

2. This protocol is in position to outgo LEACH for different or various network sizes and topologies cluster formation in LEACH, and reduces the number or quantity of data/information transmission volume through the chain of information aggregation.

3. The energy load is distributed uniformly within the network. To prevent the subsequent early death of sensor node, all sensor nodes act as leader successively.

\section{Problems in PEGASIS protocol are:}

1. In PEGASIS sensor nodes usually or probably die early.

2. It is assumed that every sensor node are often able to communicate with sink directly, however nodes typically use multi-hop communication with the sink in practical cases. Moreover, long-range communication directly from the node to the sink will breed an excessive amount of energy consumption.

3. The communication manner suffers from excessive delays caused by the one or single chain for distant nodes and a high probability for any node to become a bottleneck. 
4. It is a troublesome task for all nodes to maintain a complete database about the location of all other nodes within the network, moreover the network is not very scalable as all nodes should have global knowledge of the network and use the greedy rule or algorithm.

\section{TEEN}

In 2001, A. Manjeshwar and D. P. Agarwal [7] projected Threshold sensitive Energy Efficient sensor Network Protocol (TEEN) protocol. Nearer nodes form clusters, with a cluster heads to transmit the collected information to one higher layer. Forming the clusters, cluster heads broadcast 2 threshold values. 1st one is hard threshold; it is minimum possible value of an attribute to trigger a sensor node. Hard threshold permits nodes transmit the event, if the event happens within the range of interest. Thus a significant reduction of the transmission delay happens. Unless an amendment of minimum soft threshold happens, the node doesn't send a new data packet. Using soft threshold prevents from the redundant information/data transmission. Since the protocol is to be attentive to the rapid changes in the perceived attribute; therefore, it is appropriate for time-critical applications.

\section{Advantages in TEEN protocol are:}

1. Supported by the thresholds, data transmission are often controlled commendably, i.e., only the sensitive data we have a tendency to demand are often transmitted, so that it reduces the energy transmission consumption and improves the effectiveness and utility of the receiving data.

2. TEEN is complement for reacting to huge changes in the sensed attributes that is appropriate for reactive scenes and time decisive applications.

\section{Disadvantages in TEEN protocol are:}

1. It is not appropriate for periodic reports applications since the user might not get any data at all if the values of the attributes may not arrive at the threshold.

2. If CHs don't seem to be within the communication range of each other, the data may be vanished, because information transmission is accomplished only at CHs.

\section{APTEEN}

A. Manjeshwar and D. P. Agarwal [8] projected Adaptive Threshold sensitive Energy Efficient sensor Network Protocol (APTEEN) protocol in 2002. The protocol is a modification of TEEN aiming to capture timecritical events and periodic data collections together. The network architecture is same as TEEN. While forming clusters, the cluster heads circulate attributes, the threshold values, and therefore the transmission schedule to any or all nodes. Cluster heads are also responsible for data aggregation so as to decrease the size of data transmitted and the energy consumed. According to energy dissipation and network lifetime, TEEN provides higher performance than LEACH and APTEEN because of the reduced number of transmissions. The main shortcomings of TEEN and APTEEN are overhead and complexity of forming clusters in multiple levels, implementing threshold-based function etc. APTEEN is based on query system which permits 3 types of queries: historical, on-time, and persistent which can be employed in hybrid network.

\section{Advantages:}

1. APTEEN merges both proactive policies, which are same as that of LEACH, and reactive policies, which is same as that of TEEN. Subsequently, it is appropriate in each proactive and reactive application.

2. It embodies lots of flexibilities and set the count-time interval and the threshold values for the energy consumption by changing the count as well as the threshold values.

\section{Disadvantages:}

1. There exist supplementary complexity which is required to implement threshold functions and the count time.

2. Actually, each TEEN and APTEEN has the identical drawbacks of additional overhead and complexity of cluster construction in multiple levels.

\section{SEP}

In 2004, G. Smaragdakis, I. Matta and A. Bestavros [9] projected Stable Election Protocol (SEP) protocol. This protocol is also a further modification to the LEACH protocol. It's heterogeneous aware protocol, supported weighted election probabilities of every node to become cluster head according to their specific energy. This approach certifies that the cluster head election is arbitrarily selected and distributed based on the fraction of energy of every node assuring a uniform use of the nodes energy. In this protocol, 2 types of nodes (two tier inclustering) and 2 level hierarchies were considered.

Advantage of SEP:

1. SEP does not require any universal knowledge of energy at each election round. 


\section{Disadvantages of SEP:}

1. The shortcoming of SEP method is that the election of the cluster heads among the two type of nodes is not dynamic, which results that the nodes that are far away from the powerful nodes will die first.

\section{EECS}

In 2005, M. Ye, C. Li, G. Chen and J. Wu [10] projected Energy Efficient Clustering Scheme (EECS) protocol. It is a novel clustering scheme for periodical data collecting applications for wireless sensor networks. It elects cluster heads with more remaining energy through local radio communication. In the cluster head election phase, a stable number of candidate nodes are elected and compete for cluster heads according to the node residual energy. The competition method is localized and without iteration. The process also produces a near uniform distribution of cluster heads. Moreover in the cluster formation phase, a unique approach is introduced to balance the load among cluster heads. However, on the other hand, it will increase the necessity of global knowledge regarding the distances between the cluster-heads and the base station.

\section{Advantages}

1. EECS builds balancing point between intra-cluster energy consumption and inter-cluster communication load based on energy and distance

2. Clustering is performed by dynamic sizing based on cluster distance from the BS. This addresses the problem that the clusters with a large distance to the BS require more energy for transmission than those with a shorter distance, and produce low message overhead and uniform distribution of $\mathrm{CHs}$ compared to LEACH.

\section{Disadvantages}

1. On account of single-hop communication in EECS, long-range transmissions directly from CHs to the BS may lead to huge energy consumption. Thus, it is not suitable for large-range networks.

2. EECS needs huge global knowledge about the distances between the CHs and BS, and therefore the task of the global data aggregation adds overheads to all sensor nodes.

3. EECS produces rather more control overhead complexity because all nodes must compete for becoming $\mathrm{CHs}$.

\section{DEEC}

In 2006, Q. Li, Z. Qingxin and W. Mingwen [11] projected Distributed Energy Efficient Clustering Protocol (DEEC) protocol. DEEC protocol is a cluster based method for multi level and 2 level energy heterogeneous wireless sensor networks. In this scheme, the cluster heads are chosen using the probability based on the ratio between residual energy of every node and the average energy of the network. The era of being cluster-heads for nodes are entirely different according to their initial and residual energy. The nodes with more initial and remaining energy have greater chances of the becoming cluster heads compared to nodes with low energy.

\section{Advantages of DEEC:}

1. DEEC doesn't need any universal knowledge of energy at each election round.

2. In contrast to SEP and LEACH, DEEC will perform well in multi-level heterogeneous wireless network

\section{Disadvantages of DEEC:}

1. Advanced nodes always punish in the DEEC, particularly when their residual energy reduced and when they come in the range of the normal nodes. During this position, the advanced nodes die rapidly than the others.

\section{HEED}

O. Younis and S. Fahmy projected [4] Hybrid Energy Efficient Distributed clustering Protocol (HEED) protocol in 2004. It extends the fundamental or the basic scheme of LEACH by using residual energy as primary parameter and network topology features such as node degree, distances to neighbours are only used as secondary parameters to shatter the tie between the candidate cluster heads, as a metric for cluster choice to attain power balancing. The clustering process is split into a number of iterations, and in every iteration nodes that are not covered by any cluster head doubles their probability of becoming a cluster head. As these energyefficient clustering protocols further enables each node to probabilistically and independently decide its role in the clustered network. Moreover they cannot guarantee optimal elected set of cluster heads.

\section{Advantages of HEED protocol are:}

1. It is a distributed clustering method that benefits from the use of the two important parameters for $\mathrm{CH}$ election.

2. Low power levels of clusters endorse an increase in spatial reuse while high power levels of clusters are needed for inter-cluster communication. This imparts uniform $\mathrm{CH}$ distribution across the network and load balancing. 
3. In a multi-hop fashion, communication between $\mathrm{CHs}$ and $\mathrm{BS}$ provides more energy conservation and scalability in contrast with the single-hop fashion, i.e long range communication directly from $\mathrm{CHs}$ to the sink, as within the LEACH protocol.

\section{Limitations with HEED protocol:}

1. Tentative $\mathrm{CHs}$ that do not become final $\mathrm{CHs}$ leave some uncovered nodes. Based on implementation of HEED, these nodes are forced to become a $\mathrm{CH}$ and these forced $\mathrm{CHs}$ may not have any member associated with them or may be in range of other $\mathrm{CHs}$. As a result, more $\mathrm{CHs}$ are generated than the expected number and this also responsible for unbalanced energy consumption in the network.

2. Similar to LEACH, the clustering in each round imposes significant overhead in the network. This overhead causes remarkable energy dissipation which results in decreasing the network lifetime.

3. HEED suffers from a subsequent overhead since it needs several iterations to form the clusters. Therefore at iteration, a lot of packets are broadcasted.

4. Some $\mathrm{CHs}$, particularly near the sink, might die earlier because these $\mathrm{CHs}$ have huge workload.

\section{H-HEED}

Harneet Kour and Ajay K. Sharma, 2010 discuss about the H-HEED protocol. This protocol is basically used in heterogeneous wireless sensor network. H-HEED protocol is employed to extend the network life [2]. The impact of heterogeneity in terms of node energy in wireless sensor network has been stated. HHEED (Heterogeneous Hybrid Energy Efficient Distributed) is the revised version of the HEED protocol in terms of non- homogeneity. Here the cluster head is chosen based on the fraction of residual energy to the utmost energy possessed by the sensor nodes. Head to head communication takes place and unlike energy levelled networks have been formed. The energy efficiency has been verified in terms of the energy needed for the transmission and reception of the data. Here the node substitution takes place in order to reenergize the network and to enhance the network life.

Advantages:

1. H-HEED protocol is superior version of HEED protocol in terms of heterogeneity.

2. It is used to extend the network lifetime; network is re-energized by node replacement technique.

Disadvantages:

1. H-HEED can't work or out perform well in the homogenous environment as DEC protocol can do so.

\section{CONCLUSION}

One of the main challenges in the design of routing protocols for WSNs is energy efficiency. The energy utilization of the sensors is dominated by data transmission and reception. Therefore, routing protocols proposed for WSNs should be as energy efficient as possible to prolong the lifetime of individual sensors, and hence the network lifetime. The protocols conferred have individual merits and shortcomings. It is required to satisfy the constraints introduced by factors such as scalability, cost, fault tolerance, environment, topology alteration, and power consumption for realization of sensor networks.

Routing in sensor networks is a new research area, with a restricted but quickly emerging set of results. In this paper, hierarchical based techniques have special advantage of scalability and efficient communication. The hierarchical routing maintains the energy consumption of sensor nodes and performs data aggregation which helps in decreasing the number of transmitted messages to base station. Therefore we have concluded that hierarchical routing protocols are more efficient than the location based routing protocols in maintaining energy consumption and making more energy efficient WSNs.

\section{REFERENCES}

[1] Wiley, 2010] Fundamentals of Wireless Sensor Networks - Theory and Practice

[2] Harneet Kour and Ajay K. Sharma, "Hybrid Energy Efficient Distributed Protocol for Heterogeneous Wireless Sensor Network", International Journal of Computer Applications (0975 - 8887) Volume 4 No.6, July 2010.

[3] I.F. Akyildiz, W. Su*, Y. Sankarasubramaniam, E. Cayirci, "Wireless sensor networks: a Survey", Broadband and Wireless Networking Laboratory, School of Electrical and Computer Engineering, Georgia Institute of Technology, Atlanta, GA 30332, USA.

[4] Ossama Younis and Sonia Fahmy. 2004. Distributed Clustering in Ad-hoc Sensor Networks: A Hybrid, EnergyEfficient Approach. In Proceedings of IEEE INFOCOM, Hong Kong, an extended version appeared in IEEE Transactions on Mobile Computing, 3(4) 
[5] W. Heinzelman, A. Chandrakasan and H. Balakrishnan. 2000. Energy-Efficient Communication Protocol for Wireless Microsensor Networks. Proceedings of the $33^{\text {rd }}$ Hawaii International Conference on System Sciences (HICSS '00).

[6] S. Lindsey, C. Raghavendra. 2002. PEGASIS: Power Efficient Gathering in Sensor Information Systems. IEEE Aerospace Conference Proceedings, Vol. 3, 9-16 pp. 11251130.

[7] A. Manjeshwar and D. P. Agarwal. 2001. TEEN: a routing protocol for enhanced efficiency in wireless sensor networks. In 1st International Workshop on Parallel and Distributed Computing Issues in Wireless Networks and Mobile Computing.

[8] A. Manjeshwar and D. P. Agarwal. 2002. APTEEN: A hybrid protocol for efficient routing and comprehensive information retrieval in wireless sensor networks. Parallel and Distributed Processing Symposium, Proceedings International, IPDPS, pp. 195-202.

[9] G. Smaragdakis, I. Matta, A. Bestavros. 2004. SEP: A Stable Election Protocol for clustered heterogeneous wireless sensor networks. In Second International Workshop on Sensor and Actor Network Protocols and Applications (SANPA).

[10] M. Ye, C. Li, G. Chen, J. Wu. 2005. EECS: an energy efficient cluster scheme in wireless sensor networks. In IEEE International Workshop on Strategies for Energy Efficiency in Ad Hoc and Sensor Networks (IEEE IWSEEASN-2005), Phoenix, Arizona, April 7-9.

[11] Q. Li, Z. Qingxin, and W. Mingwen. 2006. Design of a distributed energy efficient clustering algorithm for heterogeneous wireless sensor networks. Computer Communications, vol. 29, pp. 2230-7.

[12] K. Padmanabhan, Dr. P. Kamalakkannan "Energy Efficient Adaptive Protocol for Clustered Wireless Sensor Networks" IJCSI International Journal of Computer Science Issues, Vol. 8, Issue 5, No 1, September 2011 ISSN (Online): 1694-0814 